\title{
Cough, Aortobronchial Fistula, and Air Migration in the Remnant Aneurysm Sac: An Unforeseen Path?
}

\author{
Kilsoo Yie, MD
}

The pressure gap between the aortic lumen and bronchial air spaces makes hemoptysis the most frequent feature of aortobronchial fistula (ABF). However, air migration can be a rare cause of $A B F$. The literature has demonstrated some "indirect routes" that would serve as a bridge between ABF and consecutive air migrations. However, evidence suggesting the presence of a "direct route" between them is lacking. We present an extremely rare case of newly onset cough-induced "perigraft space ABF" and ABF-related air migration in the remnant aneurysm sac occurring 3 years after an aortic surgery and a near-misdiagnosis of a delayed graft infection.

Keywords: coughing, aortobronchial fistula, air embolism

\section{Introduction}

Cough is a physiological phenomenon associated with various complications, including those of cardiovascular and respiratory systems. Although the pathophysiology of cough-related air migration is under investigation, evidence suggesting their direct association remains unclear. ${ }^{1-3)}$ In this study, we present an extremely rare case of cough-induced intra-aortic perigraft space air and discuss its mechanism and clinical significance.

\section{Jeju Soo Cardio Vascular Center (JSCVC), Jeju, South Korea}

Received: October 17, 2019; Accepted: November 27, 2019 Corresponding author: Kilsoo Yie, MD. Jeju Soo CardioVascular Center (JSCVC), 407 Nohyungro, Jeju 690-766, South Korea Tel: +82-10-2595-2185, Fax: +82-64-746-2185

E-mail: kilsooyie@gmail.com

(cc) BY-NC-SA (92020 The Editorial Committee of Annals of Vascular Diseases. This article is distributed under the terms of the Creative Commons Attribution License, which permits use, distribution, and reproduction in any medium, provided the credit of the original work, a link to the license, and indication of any change are properly given, and the original work is not used for commercial purposes. Remixed or transformed contributions must be distributed under the same license as the original.

\section{Case Report}

A 75-year-old male patient presented to the outpatient department of our hospital with excessive cough and bloody sputum for 20 days. He was an ex-smoker, with a history of 30 pack-years, and had a surgical history of aortic arch and descending thoracic aorta replacement 40 months earlier for degenerative aneurysm. The surgery for degenerative aneurysm had been conducted in two stages: total arch replacement under sternotomy, followed by descending aorta replacement via left thoracotomy using Dacron grafts (Gelweave ${ }^{\mathrm{TM}}$, Vascutek Ltd., Terumo Aortic (UK), Scotland, UK). At the end of the second operative stage, the graft had been completely wrapped by the longitudinally incised aorta. Thereafter, meticulous suturing of the aorta resulting in blood-proof status had been performed. Regular postoperative examinations had been performed for $>3$ years, with no remarkable findings (Figs. $1 \mathrm{~A}$ and 2A). The patient had also been prescribed medications for hypertension and diabetes.

In the outpatient department, the patient was febrile $\left(38.3^{\circ} \mathrm{C}\right)$ with frequent chest pain on breathing. Laboratory blood tests revealed complete blood count, chronic reactant protein, and procalcitonin levels of $6,670 \mathrm{~m} / \mathrm{mm}^{3}$, $<0.5 \mathrm{mg} / \mathrm{L}$, and $<0.5 \mu \mathrm{g} / \mathrm{L}$, respectively. Other laboratory tests results were unremarkable. However, the imaging study revealed air collection in the left hemithorax (Fig. 1B) and multiple ground-glass opacities in the lung parenchyma (Fig. 1C), which were not present in the previous imaging studies. Computed tomographic (CT) aortography showed newly onset multiple air shadows in the perigraft space, comprising an intra-aortic mural thrombus (Fig. 2B). However, there was no visible crack or hole along the suture-line of the aorta. The etiology for this peculiar finding was difficult to determine, requiring a multidisciplinary approach; the case was discussed in the hospital interdepartment discussion (HID) committee for further action. A possibility of perigraft infection was claimed by some physicians. Although we could not perform a radionuclear imaging, laboratory findings of unchanged pleural fluid amount and perigraft component 


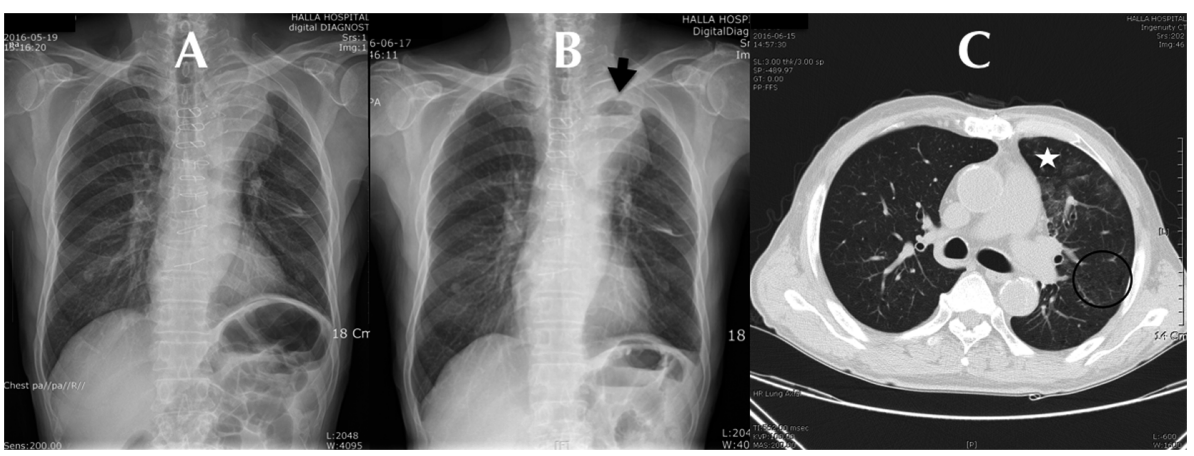

Fig. 1 A, B: Changes between pre- and post-coughing event. The black arrow indicates newly onset air density in the mediastinum. C: High-resolution computed tomography showing emphysematous appearance of pulmonary parenchyme (black circle) and aspirated blood-related ground-glass attenuation (white star).

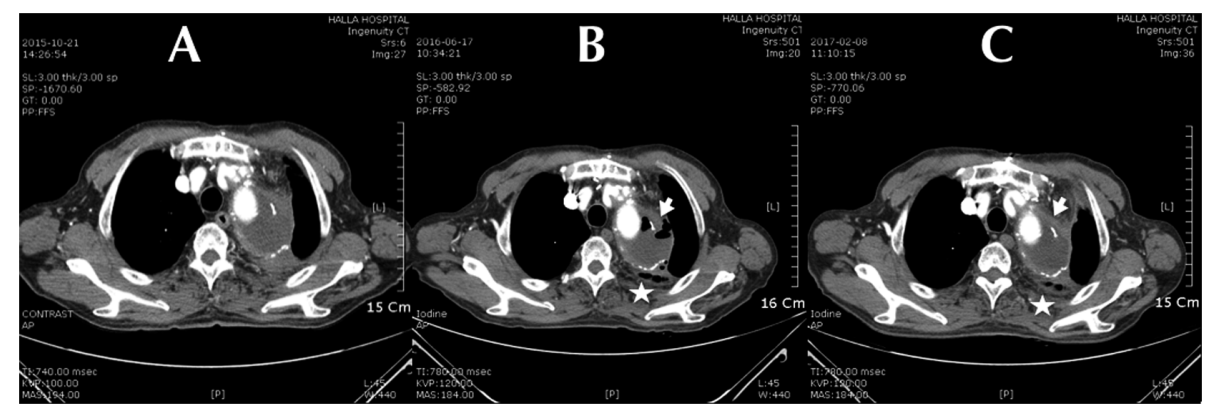

Fig. 2 Serial findings of the mediastinal structure change.

A: 8 months before the onset of cough. B: A few days later after 20-days of coughing. Note the newly onset air shadow in the intra-aortic perigraft space (white arrow) and pleural cavity (white star). C: The 6-month follow-up image. Complete resolution of aortic perigraft space air is observed.

density lowered the possibility of the graft infection. The committee concluded that severe cough of unknown etiology could have destroyed the pulmonary structures, and through the damaged bronchovascular border, pressurized bronchial air could have caused aortobronchial fistula $(\mathrm{ABF})$ and pleural cavity air. Regular observation alone, without the use of antibiotics, was recommended. Febrile sense and cough with bloody sputum disappeared after treatment with an oral nonsteroidal anti-inflammatory drug for five days. The 6-month follow-up aorta CT angiography revealed complete resolution of the air shadow in the perigraft space without any other deformation (Fig. $2 \mathrm{C})$. All laboratory values were within the reference range during the entire follow-up period.

\section{Discussion}

This case provides a few important insights. First, to the best of our knowledge, this is the first case demonstrating that cough itself can cause ABF without any other aggravating condition. Some of the common cardiovascular complications originating from vigorous cough are vasovagal hypotension, arrhythmia, rupture of small size vasculature, and dislodgment of intravascular catheters. ${ }^{4)}$ Although cough-related ABFs are rare, they frequently occur under specific co-preconditions such as pulmonary contusion and mechanical ventilation. ${ }^{1-3)}$ Because our patient had a longstanding history of smoking, a certain degree of pulmonary parenchymal destruction is obvious, leading to increased vulnerability to barotrauma. Cough, by itself, can create enough pressure to cause ABF. During heavy coughing, intrathoracic pressures of up to $300 \mathrm{mmHg}$ and expiratory velocities of up to $1,000 \mathrm{~km} / \mathrm{h}$ may be generated. ${ }^{4}$

Second, a "direct route" is assumed to be present between $\mathrm{ABF}$ and systemic arterial air migration. In our patient, the trapped air noted in the intra-aortic perigraft space could be the basis for this interpretation. Previous studies have postulated that several probable "indirect pathways" of a fistula may result in air migration. Because ABFs presenting air migration without hemoptysis have been reported only under the circumstances of increased positive airway pressure (mechanical ventilation or cardiopulmonary compression) with significant pulmonary destruction (infective irritation or cancer invasion on pulmonary vasculature), ${ }^{1-3,5)}$ we inferred that air could 
have first occurred in the low-pressure pulmonary venous circulation, not directly into the aorta, moved to the left atrium, and then to the systemic circulation. Another explanation is that air could have entered through a bridging structure, such as the patent ductus arteriosus. ${ }^{6}{ }^{6}$ However, the hypothesis "bronchial air can directly pass through the aorta" was denied because massive hemoptysis because of the pressure gap between the aorta and alveolar space must have been a predominant manifestation, if the hypothesis was indeed true. In our patient, trapped air in the intra-aortic space was filled with thrombus, not with real blood flow generating blood pressure. It would be possible to reveal fossilized air, which might be evidence of a "direct route," with the sole presentation of bloody sputum, instead of life-threatening hemoptysis. Air transportation through the suture line might have been another possibility, but it was determined to be nearly impossible because the initial blood-proof-secured suture with $>3$ years of integrity might have sufficient anastomosis tightness to keep it air-proof. Another possibility of a direct connection between the bronchial tree or a bulla and the degenerated aortic aneurysmal wall existed. However, considering the circumstances of the presenting case, disappearance of air shadow from the aneurysmal space was ruled out. Lung ventilation scintigraphy or bronchography may assist in confirming the communication between the airway and the aneurysmal sac.

Third, ABF and structural air sign, especially when they appear with perivascular tissue destruction and inflammation, may lead to an incorrect diagnosis. We almost misinterpreted the trapped air in the perigraft space, which was associated with febrility, as a sign of delayed graft infection. For accurate diagnosis of delayed graft infection, a high index of suspicion is mandatory, and it is crucial to point out not only fragmentary evidence but also detailed clinical presentation, various laboratory test results, and the time of postoperative onset. ${ }^{7}$ ) We emphasize that the diagnosis and treatment modality are best determined by consultation among experts in cardiovascular surgery, infectious disease, vascular medicine, and radiology. After its inception in 2001, we have been regularly conducting the HID meetings.

\section{Conclusion}

Cough could lead to vascular air migration in certain circumstances, without hemoptysis. However, further studies are warranted for confirming the exact pathogenesis.

\section{Disclosure Statement}

Conflict of interests: none.

\section{References}

1) Doshi HK, Thankachen R, Philip MA, et al. Bronchovenous fistula: leading to fatal massive systemic air embolism during cardiopulmonary bypass. Interact Cardiovasc Thorac Surg 2005; 4: 440-1.

2) Kau T, Rabitsch E, Celedin S, et al. When coughing can cause stroke: a case-based update on cerebral air embolism complicating biopsy of the lung. Cardiovasc Intervent Radiol 2008; 31: 848-53.

3) Ulyatt DB, Judson JA, Trubuhovich RV, et al. Cerebral arterial air embolism associated with coughing on a continuous positive airway pressure circuit. Crit Care Med 1991; 19 : 985-7.

4) Irwin RS. Complications of cough: ACCP evidence-based clinical practice guidelines. Chest 2006; 129 Suppl: 54S-8S.

5) Costarangos C, Fletcher EC. Bronchogenic carcinoma, massive hemoptysis, and systemic air embolus. Chest 1986; 90: 140-1.

6) Sheng T, Yu L. Aortobronchial fistula: secondary to patent ductus arteriosus. J Formos Med Assoc 2012; 111: 584-5.

7) Wilson WR, Bower TC, Creager MA, et al. Vascular graft infections, mycotic aneurysms, and endovascular infections: a scientific statement from the American Heart Association. Circulation 2016; 134: e412-60. 